CARDIOVASCULAR MEDICINE

\title{
Early detection of cardiac involvement in patients with sarcoidosis by a non-invasive method with ultrasonic tissue characterisation
}

\author{
E Hyodo, T Hozumi, Y Takemoto, H Watanabe, T Muro, H Yamagishi, M Yoshiyama, K Takeuchi, \\ J Yoshikawa
}

Heart 2004;90:1275-1280. doi: 10.1136/hrt.2003.027763

See end of article for authors' affiliations

Correspondence to:

Dr T Hozumi, Department of Internal Medicine and Cardiology, Osaka City University School of Medicine 1-4-3 Asahimachi, Abeno-ku, Osaka, Japan, 545-8585: thozumi@med.osaka-cu. ac.jp

Accepted 16 February 2004
Objectives: To clarify the value of cycle dependent variation of myocardial integrated backscatter (CV-IB) analysis, which non-invasively measures acoustic properties of the myocardium, for early detection of cardiac involvement in patients with sarcoidosis.

Methods: The study population consisted of 22 consecutive patients with biopsy proven sarcoidosis who did not have any abnormal findings on conventional two dimensional echocardiogram. Cardiac sarcoidosis was diagnosed by radionuclide testing including thallium-201 scintigraphy, gallium-67 scintigraphy, and cardiac fluorine-18-deoxyglucose positron emission tomography. The magnitude and delay of the CV-IB were analysed in the basal mid septum and the basal mid posterior wall of the left ventricle of all patients.

Results: The patients were divided into two groups: 8 patients with cardiac involvement and 14 patients without cardiac involvement. In the basal septum, a major reduction in the magnitude (mean (SD) 1.8 (4.4) $v 6.6(1.3), p=0.012)$ and an increase in the time delay $(1.3(0.5) v 1.0(0.1), p=0.038)$ of CV-IB were observed in patients with cardiac sarcoidosis even in the absence of two dimensional echocardiographic abnormalities. The sensitivity for detecting cardiac involvement was such that the magnitude of CV-IB in the basal septum discriminated $75 \%$ of patients with cardiac sarcoidosis from those with non-cardiac sarcoidosis, whereas two dimensional echocardiographic parameters did not discriminate between these two groups.

Conclusions: The CV-IB is decreased in the basal septum in patients with cardiac sarcoidosis even in the absence of two dimensional echocardiographic abnormalities. Analysis of CV-IB may be a useful method to detect early myocardial involvement in patients with sarcoidosis.
$\mathrm{C}$ ardiac sarcoidosis is a life threatening disease because it can result in sudden death caused by cardiac arrhythmia. ${ }^{1}$ Although cardiac involvement is responsible for as many as $85 \%$ of deaths from sarcoidosis, ${ }^{2}$ unfortunately it remains difficult to diagnose before death. ${ }^{1}$ Even considering the dramatic effect of early corticosteroid administration, the difficulty in diagnosis of this disease particularly in the early stage challenges improving the prognosis of cardiac sarcoidosis.

The two dimensional echocardiographic features typical for cardiac sarcoidosis are abnormal septal thickening or thinning, dilatation of the left ventricle, and systolic dysfunction of the left ventricle. ${ }^{4}$ However, these findings are based on permanent myocardial derangements that are usually difficult to alleviate even with corticosteroids. Therefore, early detection of cardiac sarcoidosis before these morphological abnormalities develop is essential for treatment.

Recently, ultrasonic tissue characterisation with integrated backscatter (IB) has successfully differentiated damaged myocardium from normal myocardium. Various degrees of cardiac disorder combined with myocardial tissue damage can be clearly detected with IB..$^{5-7}$ From the histological viewpoint, cardiac sarcoidosis is a form of progressive myocardial injury that results in fibrosis. Although this gradual change is strongly suspected to be detectable by ultrasonic tissue characterisation, no data exist regarding this issue. The purpose of this study was to clarify the value of IB analysis for the early diagnosis of myocardial involvement, especially before development of two dimensional echocardiographic abnormalities, in patients with sarcoidosis.

\section{METHODS}

\section{Study population}

We studied 36 consecutive patients with systemic sarcoidosis that was diagnosed histologically in extracardiac lesions (fig 1). All the patients underwent conventional two dimensional echocardiography. Ten patients with abnormal two dimensional echocardiogram were excluded because the cardiac sarcoidosis could be diagnosed from this finding. One patient with significant valvar heart disease, one with inadequate echocardiographic quality, and two with arrhythmia were also excluded from this study. The remaining 22 patients (six men, mean (SD) age 56 (11) years) who did not have any abnormal findings on two dimensional echocardiogram were enrolled into this study. At the time of medical consultation, seven patients had exertional dyspnoea, seven had chronic cough, six had blurred vision, and two had no symptoms. No patients had a history of ischaemic heart disease or evidence of previous infarction.

\section{Protocol}

We obtained an ECG, chest radiograph, measurements of serum angiotensin converting enzyme, conventional two dimensional echocardiogram, Doppler flow echocardiogram, and IB examination from all patients. Then, within seven days after echocardiography, we performed radionuclide testing, such as gallium-67 scintigraphy and cardiac

Abbreviations: ${ }^{18} \mathrm{FDG}$ PET, fluorine-18-deoxyglucose positron emission tomography; IB, integrated backscatter; CV-IB, cycle dependent variation of myocardial integrated backscatter 


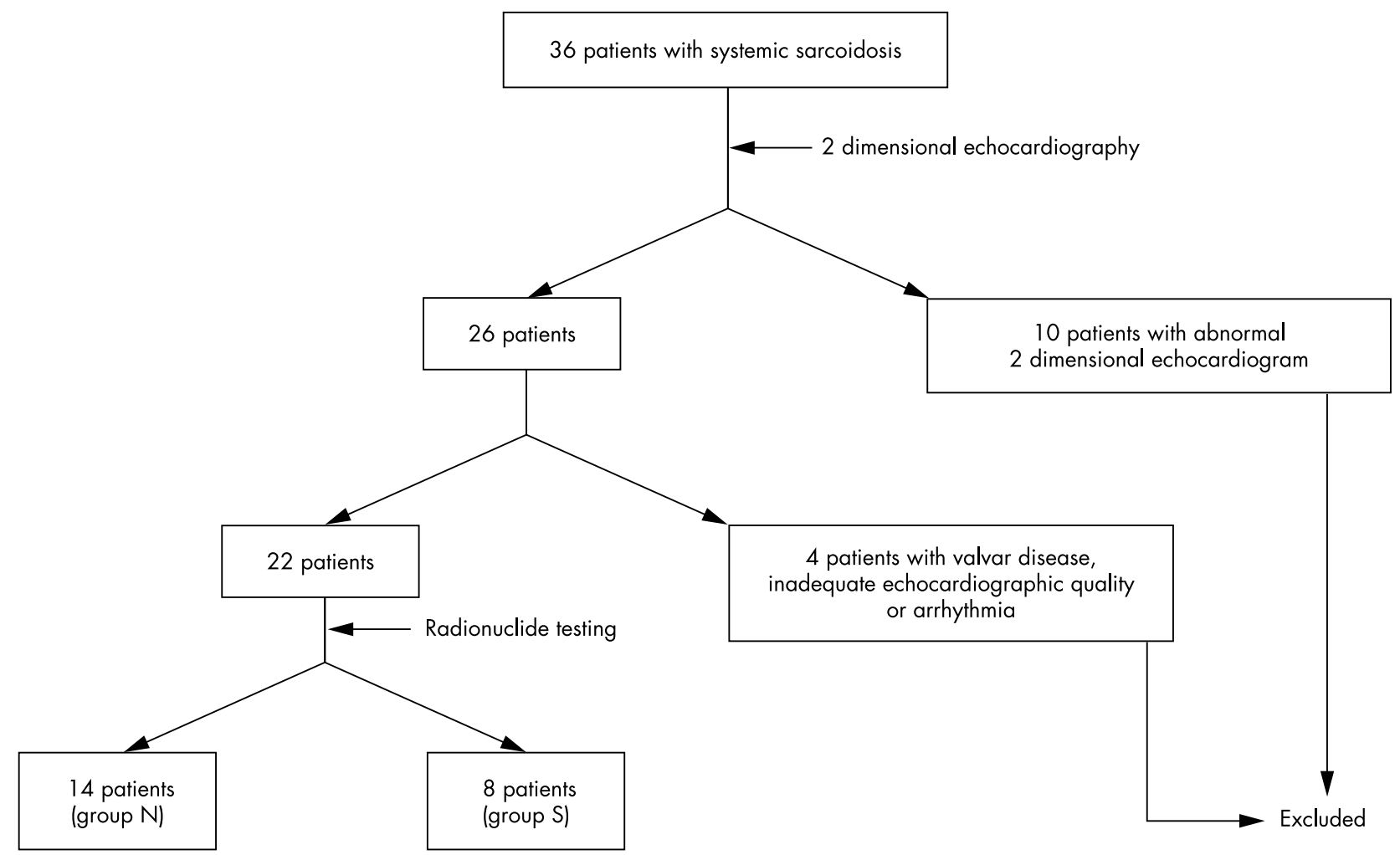

Figure 1 Patient flow chart; group $\mathrm{N}$ had no cardiac involvement, group S had cardiac involvement.

fluorine-18-deoxyglucose positron emission tomography $\left({ }^{18}\right.$ FDG PET). Thallium-201 scintigraphy was also performed when possible.

Cardiac sarcoidosis was diagnosed according to radionuclide testing as follows. ${ }^{3-12}$ One or more of the following items were required for diagnosing cardiac sarcoidosis:

- perfusion defect on ${ }^{201} \mathrm{Tl}$ single photon emission computed tomography

- abnormal accumulation on ${ }^{67} \mathrm{Ga}$ scintigraphy in the heart

- cardiac fluorine-18-deoxyglucose uptake in local segments that was higher than a normal control segment on positron emission tomography.

Two experienced nuclear cardiologists visually interpreted ${ }^{201} \mathrm{Tl}$ defects and fluorine-18-deoxyglucose uptakes. Experienced nuclear radiologists visually interpreted ${ }^{67} \mathrm{Ga}$ accumulation and were blinded to the findings of IB analysis. All patients gave informed consent to participate in this study. The study protocol was approved by the hospital ethics committee.

\section{Two dimensional echocardiography}

Echocardiographic studies of all participants were performed with an ultrasound machine (Sonos 5500 system, Philips Medical Systems, Andover, Massachusetts, USA) by two experienced investigators who did not know the patients' clinical information. Standard two dimensional echocardiogram of the left ventricle and measurement of left atrial size were from the parasternal views. Right atrial size was measured from the apical four chamber view. Ejection fraction was calculated by the modified Simpson method. ${ }^{13}$ To assess regional left ventricular contraction, regional wall thickening was calculated as: wall thickening $(\%)=($ end systolic wall thickness) - (end diastolic wall thickness)/end diastolic wall thickness $\times 100$.

\section{Acquisition and analysis of IB data}

We used a special software package (Acoustic Densitometry, Philips Medical Systems) incorporated into the Sonos 5500 system (version B) with a wideband sector transducer (S4). Sixty frames from consecutive cardiac cycles (30 frames/s) are displayed after scan conversion and were stored on the optical disc. The dynamic range of the IB images was $64 \mathrm{~dB}$. We used multiple segmental analyses. ${ }^{7}$ The time gain compensation was adjusted to give equal gain at each depth of the left ventricle in each patient. The IB amplitude was measured from the cine loop of the parasternal long axis view of the left ventricle (fig 2) by the experienced investigator, who was unaware of patients' information. An elliptical region of interest, of $31 \times 31$ or $41 \times 41$ pixels, was placed in either the mid or basal interventricular septum or in those portions of the posterior wall of the left ventricle. In each case, we determined as large as possible a region of interest so that the region of interest could be placed at the mid portion between the endocardial and epicardial borders of the myocardium. We manually adjusted the position of the region of interest in each frame to avoid specular reflections from interfaces between endocardium and blood. From the time-intensity waveforms, the magnitude of cycle dependent variation of myocardial IB (CV-IB) was calculated as the difference between the minimum and maximum values in a cardiac cycle, averaged for at least two consecutive beats (fig 2).

Because regional contraction in the affected area may not necessarily be synchronised exactly with global contractile events, we calculated the time delay of CV-IB, which was defined as the time interval between the onset of the $\mathrm{R}$ wave on the ECG to the nadir of the IB curve divided by the QT interval (fig 2). ${ }^{67}$ Because each stored cine loop consisted of 60 frames from consecutive cardiac cycles, the time delay of CV-IB could be obtained from the same cardiac cycle by determining the ratio of frame numbers from the $\mathrm{R}$ wave to 

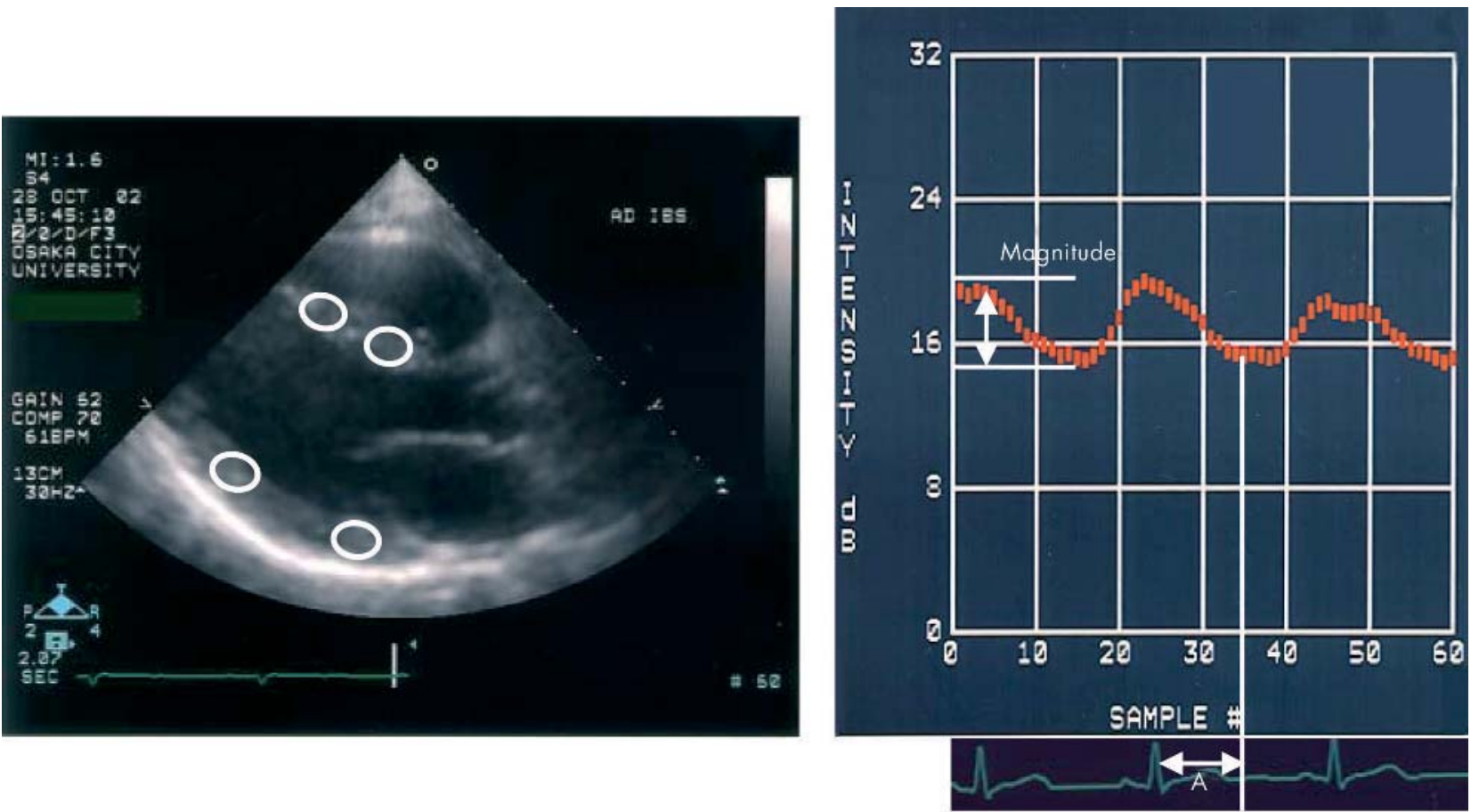

Delay=A/QT interval

Figure 2 Parasternal long axis integrated backscatter (IB) imaging interfaced with acoustic densitometry (left) and a representative curve of cycle dependent variation of myocardial IB (CV-IB) (right). An elliptical region of interest was placed in the mid or basal myocardial segment of the interventricular septum and posterior wall.

the nadir of the IB curve and the ratio from the Q wave to end of the $\mathrm{T}$ wave of the ECG. In this study, we used the phase corrected magnitude. ${ }^{6}$ If the time delay of CV-IB was $\leqslant 1.2$, the magnitude of CV-IB was multiplied by 1.0 (synchronous pattern); if the time delay was $>1.2$, the magnitude of CV-IB was multiplied by -1.0 (asynchronous pattern). ${ }^{6}$

\section{Reproducibility of data}

Interobserver and intraobserver variabilities were assessed for CV-IB measurements in 10 randomly selected patients. Interobserver variability was calculated as the standard deviation of the difference between the measurements of two independent observers who were unaware of the other patient data and was expressed as a percentage of the average value. Intraobserver variability was calculated as the standard deviation of the difference between the first and second determination (two week interval) for a single observer and was expressed as a percentage of the average value.

The mean absolute differences in the magnitude of CV-IB were 4.0 (3.8)\% (intraobserver) and 3.8 (3.5)\% (interobserver). The mean differences in the time delay of CV-IB were 5.1 (4.8)\% (intraobserver) and 5.3 (5.9)\% (interobserver).

\section{Statistical analysis}

Values are expressed as mean (SD). Differences between the two groups were assessed with the $\chi^{2}$ test or Fisher's exact test for categorical variables. Continuous variables were compared by the unpaired Student's $t$ test. Differences were considered significant at $\mathrm{p}<0.05$.

\section{RESULTS}

Of the 22 patients, 14 (group N) had no feature of cardiac involvement and eight had cardiac involvement (group S) according to the criteria described above (fig 1). Table 1 shows the clinical characteristics of the patients. There were no differences between the two groups regarding baseline characteristics. The prevalence of ${ }^{201} \mathrm{Tl}$ scintigraphy and cardiac ${ }^{18}$ FDG PET abnormality was significantly greater in group $\mathrm{S}$ than in group $\mathrm{N}$.

\section{IB and two dimensional echocardiography}

No echocardiographic or Doppler features differentiated group $\mathrm{S}$ from group $\mathrm{N}$ (table 2). There was no right ventricular wall motion abnormality in patients of group $\mathrm{S}$. Table 3 shows the phase corrected magnitude and time delay of CV-IB in the mid and basal interventricular septum and in those portions of the posterior wall of the left ventricle. In contrast to two dimensional echocardiography, which could not differentiate between the groups, there was a significant decrease in the phase corrected magnitude $(p=0.012)$ and increase in the time delay $(\mathrm{p}=0.038)$ of CV-IB in the basal interventricular septum in group $\mathrm{S}$ (fig 3 ).

Table 1 Clinical characteristics

\begin{tabular}{lll}
\hline Variable & $\begin{array}{l}\text { Group N } \\
(\mathbf{n}=14)\end{array}$ & $\begin{array}{l}\text { Group S } \\
(\mathbf{n}=8)\end{array}$ \\
\hline Age (years) & $57(13)$ & $54(10)$ \\
Body mass index $\left(\mathrm{kg} / \mathrm{m}^{2}\right)$ & $23.6(2.2)$ & $23.0(4.0)$ \\
Systolic BP $(\mathrm{mm} \mathrm{Hg})$ & $121(9)$ & $124(10)$ \\
Diastolic BP $(\mathrm{mm} \mathrm{Hg})$ & $64(7)$ & $67(9)$ \\
ACE activity (IU/I) & $32.1(4.0)$ & $31.1(10.1)$ \\
Men/women & $4 / 10$ & $2 / 6$ \\
Chest radiograph abnormality & 11 & 8 \\
${ }^{201}$ Tl scintigraphy abnormality & $0(\mathrm{n}=9)$ & $3^{*}(\mathrm{n}=5)$ \\
${ }^{18} \mathrm{FDG}$ PET abnormality & 0 & $8^{*}$ \\
${ }^{67}$ Ga scintigram abnormality & 11 & 7 \\
Extracardiac & 0 & 1 \\
Intracardiac & &
\end{tabular}

Data are expressed as mean (SD) or number of patients. ${ }^{*} \mathrm{p}<0.05 v$ group $\mathrm{N}$.

$\mathrm{ACE}$, angiotensin converting enzyme; $\mathrm{BP}$, blood pressure; ${ }^{18} \mathrm{FDG} \mathrm{PET}$, fluorine-18-deoxyglucose positron emission tomography. 
In examining the sensitivity of this test in the basal interventricular septum in discriminating patients with cardiac sarcoidosis from patients with non-cardiac sarcoidosis through individual analysis, we found that the magnitude of CV-IB in the basal interventricular septum was able to discriminate $75 \%$ (six of eight; cut off value 4.7 ) of patients with cardiac sarcoidosis from patients with non-cardiac sarcoidosis, whereas two dimensional echocardiographic parameters did not discriminate between these two groups.

\section{DISCUSSION}

In this study, we showed for the first time that non-invasive CV-IB in the basal interventricular septum can be useful for early diagnosis of cardiac involvement in patients with sarcoidosis.

It has been difficult to diagnose cardiac sarcoidosis, particularly in the early stage, by conventional methods. Two dimensional echocardiography is widely used to detect cardiac involvement. The two dimensional echocardiographic features typical for cardiac sarcoidosis are abnormal septal thickening or thinning, dilatation of the left ventricle, and systolic dysfunction of the left ventricle. ${ }^{4}$ However, these findings are based on permanent myocardial derangements that are usually difficult to alleviate even with corticosteroids. Therefore, two dimensional echocardiography may not be sensitive enough to detect small abnormalities, which may occur in the early stage of cardiac involvement. ${ }^{4}$

Although ${ }^{201} \mathrm{Tl}$ and ${ }^{67} \mathrm{Ga}$ scintigraphy have also been used, they have limited value in diagnosis because of reduced sensitivity and specificity, inadequate spatial resolution, radiation exposure, cost, and time. Imaging with cardiac ${ }^{18}$ FDG PET, which assesses disease activity at the cellular level, would make it more sensitive and specific than other activity markers. ${ }^{10-12}$ However, at present, its routine use is unwarranted because of its limited availability and high cost.

In this study, we first observed a major reduction in the magnitude and a significant increase in the time delay of CVIB in the basal interventricular septum in patients with cardiac sarcoidosis. Several mechanisms may explain why the magnitude of CV-IB decreases in patients with cardiac sarcoidosis: firstly, decreased regional myocardial contractile function; secondly, altered myocardial acoustic properties with the increased accumulation of fibrosis; and thirdly, altered myocardial acoustic properties influenced by myocytolysis and cell infiltration in the myocardium. Reduced regional myocardial contractile function may play a part in

Table 2 Echocardiographic indexes

\begin{tabular}{lll}
\hline Variable & $\begin{array}{l}\text { Group N } \\
(\mathbf{n}=14)\end{array}$ & $\begin{array}{l}\text { Group S } \\
(\mathbf{n}=8)\end{array}$ \\
\hline LVDd (mm) & $44(4)$ & $46(7)$ \\
LVDs (mm) & $24(4)$ & $26(7)$ \\
LVEF (\%) & $64(4)$ & $63(4)$ \\
LAD (mm) & $37(7)$ & $36(6)$ \\
RAD (mm) & $30(5)$ & $32(4)$ \\
IVS thickness (mm) & $9(1)$ & $9(2)$ \\
PW thickness (mm) & $9(1)$ & $9(1)$ \\
Pericardial effusion & $0 / 14$ & $1 / 8$ \\
WT of the basal septum (\%) & $57(4)$ & $53(11)$ \\
TMF-E (m/s) & $0.81(0.17)$ & $0.77(0.27)$ \\
TMF-A (m/s) & $0.77(0.40)$ & $0.74(0.13)$ \\
TMF-E/A & $1.07(0.64)$ & $1.06(0.42)$ \\
TMF-E-DT (m/s) & $196(32)$ & $206(37)$ \\
\hline
\end{tabular}

Data are expressed as mean (SD)

A, peak atrial filling wave velocity; DT, deceleration time; $E$, peak early diastolic wave velocity; IVS, interventricular septum; LAD, left atrial diameter; LVDd, left ventricular end diastolic diameter; LVDs, left ventricular end systolic diameter; LVEF, left ventricular ejection fraction PW, posterior wall; RAD, right atrial diameter; TMF, transmitral flow; WT, wall thickening.
Table 3 Cycle dependent variation (phase corrected magnitude and delay) of myocardial integrated backscatter data

\begin{tabular}{lll}
\hline Variable & $\begin{array}{l}\text { Group N } \\
(\mathbf{n}=14)\end{array}$ & $\begin{array}{l}\text { Group S } \\
(\mathbf{n}=8)\end{array}$ \\
\hline Magnitude in mid-IVS (dB) & $6.6(1.2)$ & $6.0(0.6)$ \\
Magnitude in basal IVS (dB) & $6.6(1.3)$ & $1.8(4.4)^{*}$ \\
Magnitude in mid-PW (dB) & $8.4(1.4)$ & $8.4(1.3)$ \\
Magnitude in basal PW (dB) & $8.3(1.3)$ & $8.3(1.5)$ \\
Delay in mid-IVS & $1.0(0.1)$ & $1.0(0.1)$ \\
Delay in basal IVS & $1.0(0.1)$ & $1.3(0.5)^{*}$ \\
Delay in mid-PW & $0.9(0.1)$ & $0.9(0.1)$ \\
Delay in basal PW & $1.0(0.1)$ & $1.0(0.1)$ \\
\hline
\end{tabular}

Data are expressed as mean (SD).

${ }^{*} \mathrm{p}<0.05 \vee$ group $\mathrm{N}$.

the reduced CV-IB. ${ }^{14}$ However, regional contractile function assessed by percentage systolic wall thickening in the interventricular septum was not different between group $\mathrm{S}$ and group N. Previous studies reported that wall thickening is not linearly related to the magnitude of the cyclical variation in dogs with acute coronary occlusion followed by reperfusion. ${ }^{15} 16$ Although influenced by wall thickening, CVIB appears to reflect the intrinsic contraction performance rather than myocardial thickening. ${ }^{17-21}$ Fibrosis is connected to an increase in collagen and it is known that increased collagen concentrations change the acoustic properties of the myocardium $^{22}$ and reduced the magnitude of CV-IB. ${ }^{18}{ }^{21}$ Matsui and colleagues ${ }^{23}$ showed that cardiac sarcoidosis was linked to certain histological patterns, such as lymphocytic infiltration, granulomata with epithelial cells, and fibrosis. Myocytolysis and cell infiltration lead to alterations in fibre arrangement and the direction of the dominant myofibrils. Myocardial fibrils may also fade due to cell infiltration such that the originally heterogeneous medium of the myocardium becomes more homogeneous. These irreversible changes in the myocardium can partially explain these findings.

The mechanisms of the temporal delay are also unclear. Post-systolic thickening, which reflects persisting contraction or delayed relaxation, of the myocardium may be related to time delay of CV-IB in patients with cardiac sarcoidosis. In this study, we observed post-systolic events in the altered myocardium and have provided an approach for their detection and quantification in patients with cardiac sarcoidosis. Because these events were considered to be unfavourable to global contraction, we expressed the corrected magnitude of CV-IB as a negative value if the CV-IB pattern was asynchronous.

Previous studies ${ }^{2}$ showed that the septum is compromised in more than $90 \%$ of necropsy samples of tissue with cardiac sarcoidosis. In fact, seven of eight patients in group $S$ had radionuclide abnormalities in the basal anteroseptal segment in this study. In accordance with this observation, the basal interventricular septum appears to be a suitable target structure for assessment of cardiac involvement.

Ultrasonic tissue characterisation is simple, non-invasive, and applicable to many patients in the clinical setting. Analysis of CV-IB can be a new diagnostic modality for early detection of cardiac involvement in patients with sarcoidosis.

\section{Limitations}

There are several limitations in the present study. The magnitude and time delay of CV-IB depend on the angle between the fibre orientation and the ultrasonic beam. ${ }^{24}$ In this study, we placed multiple regions of interest in the interventricular septum and posterior wall to the extent that 

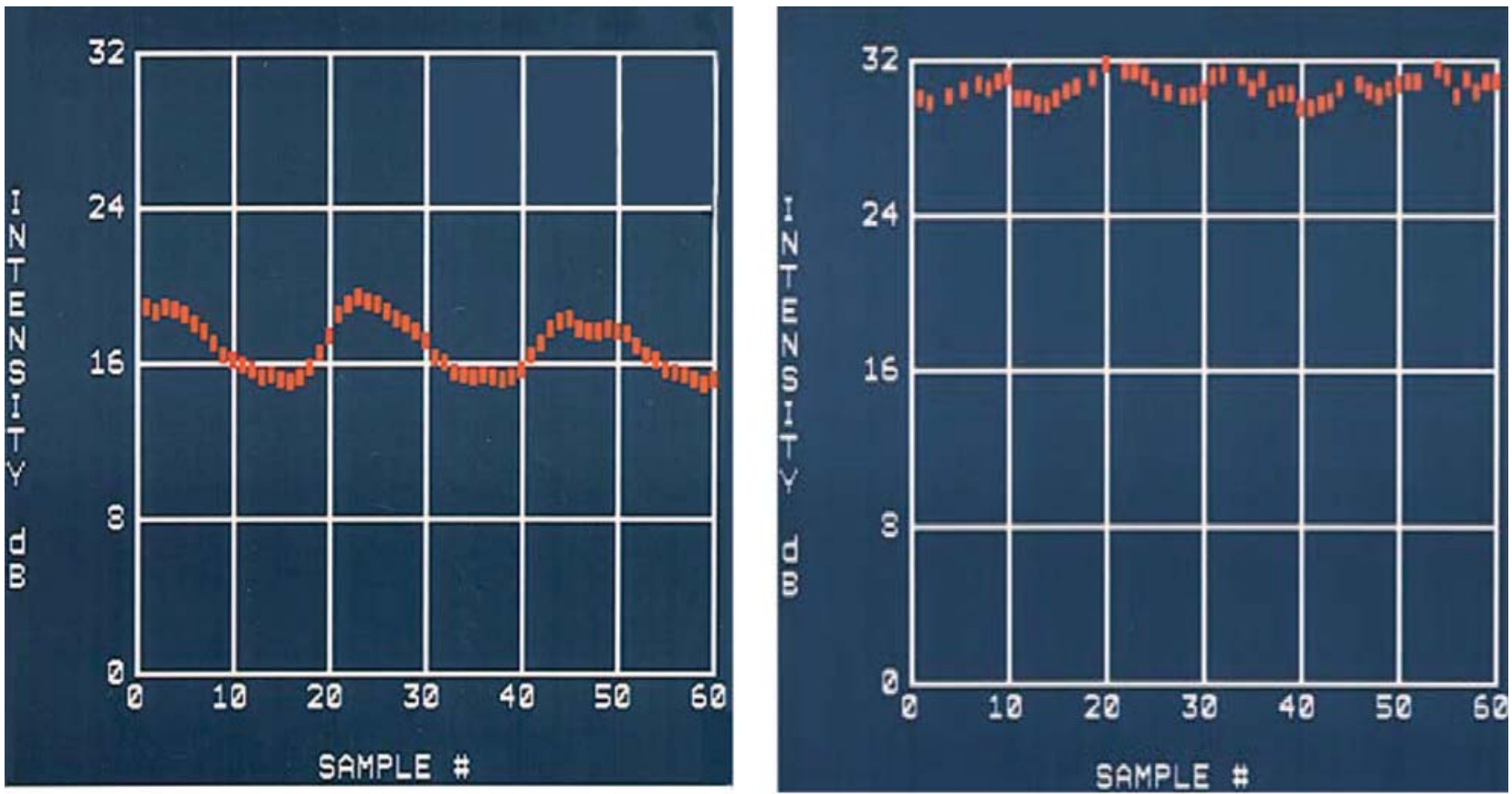

ECG

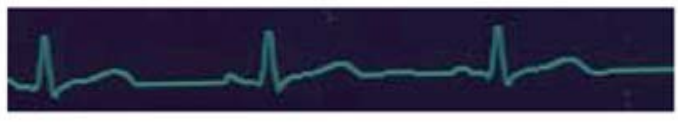

Group N

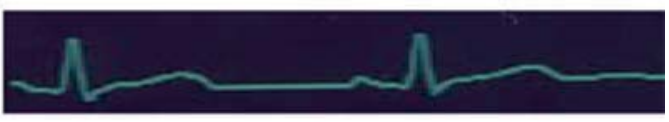

Group S

Figure 3 Representative CV-IB curves in the basal interventricular septum in patients of group N (left) and group S (right). The magnitude of CV-IB was significantly greater in the patients of group $\mathrm{N}$ than in the patients of group $\mathrm{S}$. The CV-IB pattern was synchronous in group $\mathrm{N}$ but asynchronous in group $S$.

fibre orientation was adequate, which avoided the angle problem as much as possible.

In this study, diagnosis of cardiac sarcoidosis was based on radionuclide testing, such as ${ }^{201} \mathrm{Tl}$ scintigraphy, ${ }^{67} \mathrm{Ga}$ scintigraphy, and cardiac ${ }^{18}$ FDG PET. Although detection of epithelioid granuloma in endomyocardial biopsy specimens provides a definitive diagnosis of cardiac sarcoidosis, the sensitivity of this characteristic pathological finding is relatively low..$^{25}$ Thus, we chose radionuclide abnormalities in the diagnostic criteria of cardiac sarcoidosis because recent studies have shown that cardiac involvement can be diagnosed based on radionuclide abnormalities in patients with sarcoidosis. ${ }^{38-11}$

Abnormal radionuclide findings were evaluated in all left ventricular segments for diagnosis of cardiac sarcoidosis. In IB analysis, we evaluated the basal and mid-interventricular septum because most patients with cardiac sarcoidosis had the involvement in the interventricular septum. ${ }^{2}$ This discrepancy in the evaluated lesion may result in decreasing sensitivity of diagnosing cardiac involvement. However, there were no abnormal findings in the interventricular septum by nuclear examinations in only one of eight patients (group S). This result supports the previous report that the interventricular septum is involved in most cases of cardiac sarcoidosis. We think that the analysis of CV-IB in the basal interventricular septum would be useful for detecting cardiac involvement in patients with sarcoidosis.

Radionuclide abnormalities in the myocardium may not be specific to sarcoidosis and may occur in patients with ischaemic heart disease. However, these abnormalities localised in the interventricular septum are uncommon in coronary artery disease and may be specific for cardiac sarcoidosis.

\section{Conclusion}

Analysis of CV-IB in the basal septum may be a new, noninvasive modality for early diagnosis of cardiac involvement, which enables us to administer corticosteroid in the early stage of cardiac involvement, in patients with sarcoidosis.

\section{Authors' affiliations}

E Hyodo, T Hozumi, Y Takemoto, H Watanabe, T Muro, H Yamagishi, M Yoshiyama, K Takeuchi, J Yoshikawa, Department of Internal Medicine and Cardiology, Osaka City University School of Medicine, Osaka, Japan

\section{REFERENCES}

Mitchell DN, du Bois RM, Oldershaw PJ. Cardiac sarcoidosis. BMJ 1997;314:320-1.

2 Silverman KJ, Hutchins GM, Bulkley BH. Cardiac sarcoid: a clinicopathologic study of 84 unselected patients with systemic sarcoidosis. Circulation 1978;58:1204-11.

3 Sharma OP, Maheshwari A, Thaker K. Myocardial sarcoidosis. Chest 1993;103:253-8

4 Burstow DJ, Tajik AJ, Bailey KR, et al. Two-dimensional echocardiographic findings in systemic sarcoidosis. Am J Cardiol 1989;63:478-82.

5 Masuyama T, Nellessen U, Schnittger I, et al. Ultrasonic tissue characterization with a real time integrated backscatter imaging system in normal and aging human hearts. J Am Coll Cardiol 1989;14:1702-8.

6 Takiuchi S, Ito $\mathrm{H}$, Iwakura $\mathrm{K}$, et al. Ultrasonic tissue characterization predicts myocardial viability in early stage of reperfused acute myocardial infarction. Circulation 1998;97:356-62.

7 Muro T, Ota T, Watanabe $\mathrm{H}$, et al. Prediction of contractile reserve by cyclic variation of integrated backscatter of the myocardium in patients with chronic left ventricular dysfunction. Heart 2001;85:165-70.

8 Newman LS, Rose CS, Maier LA. Sarcoidosis. N Engl J Med 1997; 336:1224-34

9 Okayama K, Kurata C, Tawarahara K, et al. Diagnostic and prognostic value of myocardial scintigraphy with thallium-201 and gallium-67 in cardiac sarcoidosis. Chest 1995; 107:330-4.

10 Okumura W, Iwasaki T, Ueda T, et al. [Usefulness of 18F-FDG PET for diagnosis of cardiac sarcoidosis]. Kaku Igaku 1999;36:341-8. 
11 Takeda N, Yokoyama I, Hiroi Y, et al. Positron emission tomography predicted recovery of complete $A-V$ nodal dysfunction in a patient with cardiac sarcoidosis. Circulation 2002; 105:1144-5.

12 Yamagishi $H$, Shirai $N$, Takagi $M$, ef al. Identification of cardiac sarcoidosis with (13)N-NH(3)/(18)F-FDG PET. J Nucl Med 2003:44:1030-6.

13 Schiller NB, Shah PM, Crawford M, et al. Recommendations for quantitation of the left ventricle by two-dimensional echocardiography. American Society of Echocardiography committee on standards, subcommittee on quantitation of two-dimensional echocardiograms. J Am Soc Echocardiogr 1989:2:358-67.

14 Wickline SA, Thomas $U$, Miller JG, et al. The dependence of myocardial ultrasonic integrated backscatter on contractile performance. Circulation 1985;72:183-92.

15 Wickline SA, Thomas $\sqcup$, Miller JG, et al. Sensitive detection of the effects of reperfusion on myocardium by ultrasonic tissue characterization with integrated backscatter. Circulation 1986;74:389-400.

16 Goens MB, Karr SS, Martin GR. Cyclic variation of integrated ultrasound backscatter: normal and abnormal myocardial patterns in children. J Am Soc Echocardiogr 1996;9:616-21.

17 Barzilai B, Madaras El, Sobel BE, et al. Effects of myocardial contraction on ultrasonic backscatter before and after ischemia. Am J Physiol 1984;247:H478-83.

18 Perez JE, McGill JB, Santiago JV, et al. Abnormal myocardial acoustic properties in diabetic patients and their correlation with the severity of disease. J Am Coll Cardiol 1992;19:1154-62.
19 Masuyama T, Valantine HA, Gibbons R, et al. Serial measurement of integrated ultrasonic backscatter in human cardiac allografts for the recognition of acute rejection. Circulation 1990;81:829-39.

20 Koyama J, Ray-Sequin PA, Falk RH. Prognostic significance of ultrasound myocardial tissue characterization in patients with cardiac amyloidosis. Circulation 2002;106:556-61.

21 Di Bello V, Giorgi D, Talini E, et al. Incremental value of ultrasonic tissue characterization (backscatter) in the evaluation of left ventricular myocardial structure and mechanics in essential arterial hypertension. Circulation 2003:107:74-80.

22 Lieback E, Hardouin I, Meyer R, et al. Clinical value of echocardiographic tissue characterization in the diagnosis of myocarditis. Eur Heart $J$ 1996; 17:135-42.

23 Matsui Y, Iwai K, Tachibana T, et al. Clinicopathological study of fatal myocardial sarcoidosis. Ann N Y Acad Sci 1976;278:455-69.

24 Holland MR, Wilkenshoff UM, Finch-Johnston $A E$, et al. Effects of myocardial fiber orientation in echocardiography: quantitative measurements and computer simulation of the regional dependence of backscattered ultrasound in the parasternal short-axis view. J Am Soc Echocardiogr 1998:11:929-37.

25 Uemura A, Morimoto S, Hiramitsu S, et al. Histologic diagnostic rate of cardiac sarcoidosis: evaluation of endomyocardial biopsies. Am Heart $J$ 1999; 138:299-302.

26 Ratner SJ, Fenoglio JJ Jr, Ursell PC. Utility of endomyocardial biopsy in the diagnosis of cardiac sarcoidosis. Chest 1986;90:528-33.

\section{IMAGES IN CARDIOLOGY}

\section{Braid-like coronary appearance by coronary angiography in systemic lupus erythematosis}

A

56 year old Japanese woman was referred to our hospital because of bradycardia and dyspnoea. She had a 30 year history of corticoid treatment and haemodialysis for systemic lupus erythematosis. Her coronary risk factors included hyperlipidaemia and diabetes mellitus two years previously. An ECG revealed first degree atrioventricular block, incomplete right bundle brunch block, and inverted T wave in leads V2-6. She was diagnosed with an anteroseptal myocardial infarction.

Cardiac catheterisation was performed at 14 days after admission. The coronary angiogram revealed no significant stenosis, however the proximal segment of the left anterior descending artery (LAD) had an approximately $5 \mathrm{~cm}$ length braid-like lesion (panel A). Angioscopy was undertaken with a 4.5 French catheter advanced proximally to the LAD through a guidewire under fluoroscopy. After equipping with an occlusive cuff for low pressure inflation, a porous vessel surrounding white smooth intima without thrombus was observed at angioscopy (panel B).

Intravascular ultrasound imaging was performed with a 2.9 French, $20 \mathrm{MHz}$ catheter in the LAD from the distal to the proximal lesion. Multiple channel formation was demonstrated in the
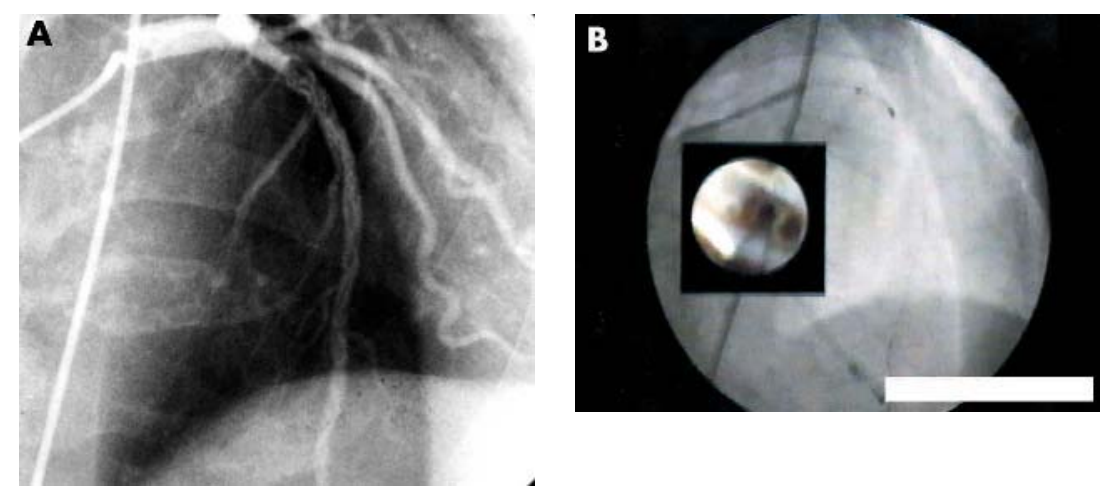

lumen of the native coronary artery lesion (panel C). The structure of the partition comprised hard tissue, including calcification.

It has been suggested that braid-like lesions are caused by thrombosis and recanalisation during the course of arteritis. This is the first report of a braid-like lesion observed by angioscopy and intravascular ultrasound imaging in a patient with systemic lupus erythematosis.

R Uemura K Mizuno ryota-uemura@mqb.biglobe.ne.jp

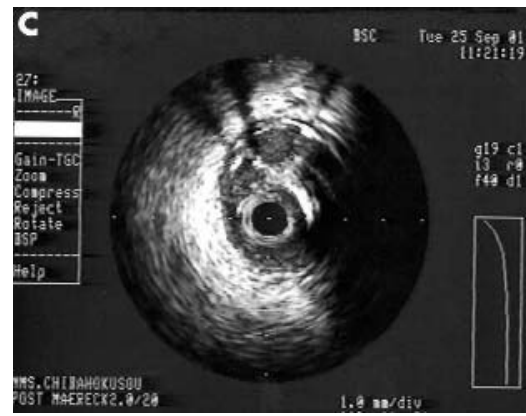

